\title{
Low Cycle and Thermo-Mechanical Fatigue of Friction Welded Dissimilar Superalloys Joint*
}

\author{
Motoki SAKAGUCHI**, Atsushi SANO**, Tra Hung TRAN **, \\ Masakazu OKAZAKI** and Masaru SEKIHARA*** \\ **Department of Mechanical Engineering, Nagaoka University of Technology \\ Kamitomioka-machi 1603-1, Nagaoka-shi, Niigata, Japan \\ E-mail:sakaguti@mech.nagaokaut.ac.jp \\ *** Hitachi Ltd., Material Research Laboratory \\ Saiwai-cho, Hitachi-shi, Ibaraki, Japan
}

\begin{abstract}
The high temperature strengths of the dissimilar friction welded superalloys joint between the cast polycrystalline Mar-M247 and the forged IN718 alloys have been investigated under low cycle and thermo-mechanical fatigue loadings, in comparison with those of the base metals. The experiments showed that the lives of the dissimilar joints were significantly influenced by the test conditions and loading modes. Not only the lives themselves but also the failure positions and mechanisms were sensitive to the loading mode. The fracture behaviors depending on the loading modes and test conditions were discussed, based on the macroscopic elastic follow-up mechanism and the microstructural inhomogeneity in the friction weld joint.
\end{abstract}

Key words: Dissimilar Metals Welding, Friction Welding, Low Cycle Fatigue, Thermo-Mechanical Fatigue, Elastic Follow-up, Failure Mechanism, Heat Affected Zone

\section{Introduction}

A friction welding is a solid state welding process in which joining can be achieved at a temperature below the melting point. This method is now recognized as a candidate technique to fabricate superalloys joint ${ }^{(1)-(6)}$, since it has generally many types of advantages; narrow heat affected zone (HAZ) without the microfissuring, no filler metals and no special requirement of surface preparation. If the hybrid dissimilar superalloys structures are successfully developed, they are expected to make a significant contribution to improve high temperature performance and thermal efficiency of gas turbines. In these hybrid structures, however, thermal stress induced by mismatches in thermal expansion coefficients and thermal conductivities between dissimilar base materials is a critical problem at high temperatures ${ }^{(7)-(10)}$. Thus, it is inevitable to know the comprehensive information on low cycle fatigue (LCF), high cycle fatigue, creep fatigue and thermo-mechanical fatigue (TMF). This knowledge can also provide some important aspects not only for the fabrication of composite structures but for the repairing technologies for the high temperature components.

The objective in this work is to investigate the high temperature fatigue strength of the dissimilar friction welded superalloys joint consisting of the cast polycrystalline Mar-M247 and the forged Inconel 718 alloys (hereinafter, the dissimilar friction welded joint will be denoted by DFW, and the two base alloys will be by M247 and IN718, respectively). Strain controlled LCF and TMF tests were carried out under various test conditions, where the 
experimental variables were strain rates, strain hold time and strain/temperature phase angle. Fatigue lives and fracture behaviors of DFW joints were discussed comparing with those of the base metals.

\section{Experimental Procedures}

\subsection{Friction welding for DFW dissimilar joint}

Dissimilar metals welded joint between the cast polycrystalline M247 and the forged IN 718 alloys is a target in this work. The chemical compositions of the base metals are shown in Table 1. The initial average grain sizes of both base metals were $5-10 \mu \mathrm{m}$ in the IN718 and $0.5-2 \mathrm{~mm}$ in $\mathrm{M} 247$, respectively. Many primary tests were carried out to fabricate an optimal DFW joint, where the fabrication variables were rotation speed of base metals, preheating time, compressive normal stress, duration time, upset pressure, upset hold time and the post weld heat treatment. A final welding condition has been determined, based on the following properties; no significant defects, no significant heat affected zone (HAZ) near the welded interface and moderately high tensile strength and ductility comparable to the base alloys. In the DFW joint finally optimized the HAZ was developed on the IN718 side by about $300 \mu \mathrm{m}$ in thickness, where the hardness was lower a little than that on the base metal side (see Fig.13).

(wt. \%)

Table1 Chemical composition of IN718 and Mar-M247.

\begin{tabular}{ccccccccccccccccc}
\hline Composition & $\mathrm{Ni}$ & $\mathrm{Cr}$ & $\mathrm{Co}$ & $\mathrm{Mo}$ & $\mathrm{W}$ & $\mathrm{Ta}$ & $\mathrm{Nb}$ & $\mathrm{Al}$ & $\mathrm{Ti}$ & $\mathrm{Fe}$ & $\mathrm{Mn}$ & $\mathrm{Si}$ & $\mathrm{C}$ & $\mathrm{B}$ & $\mathrm{Zr}$ & $\mathrm{Hf}$ \\
\hline IN 718 & 52.5 & 19 & - & 3 & - & - & 5.1 & 0.5 & 0.9 & 18.5 & 0.2 & 0.2 & 0.04 & - & - & - \\
Mar-M247 & 60 & 8.3 & 10 & 0.7 & 10 & 3 & - & 5.5 & 1 & - & - & - & 0.14 & 0.015 & 0.05 & 1.5 \\
\hline
\end{tabular}

Table2 Test conditions for LCF and TMF tests employed.

\begin{tabular}{|c|c|c|c|c|c|c|c|}
\hline Specimen & Test type & Strain wave form & Temp. $\left[{ }^{\circ} \mathrm{C}\right]$ & Strain ratio & Strain rate $[1 / \mathrm{sec}]$ & Tension hold time & Remarks \\
\hline \multirow{5}{*}{ DFW joint } & \multirow{3}{*}{ LCF } & fast-fast & \multirow{3}{*}{650} & -1 & $5 \times 10^{-4}$ & 0 & f-f LCF \\
\hline & & slow-slow & & -1 & $2 \times 10^{-4}$ & 0 & s-s LCF \\
\hline & & tension hold & & -1 & $5 \times 10^{-4}$ & $10 \mathrm{~min}$ & tension hold LCF \\
\hline & \multirow{2}{*}{ TMF } & out-of-phase & \multirow{2}{*}{$300-650$} & -1 & $2 \times 10^{-4}$ & 0 & OP TMF \\
\hline & & in-phase & & -1 & $2 \times 10^{-4}$ & 0 & IP TMF \\
\hline \multirow{2}{*}{ M247 } & \multirow{2}{*}{$\mathrm{LCF}$} & fast-fast & \multirow{2}{*}{650} & -1 & $5 \times 10^{-4}$ & 0 & f-f LCF \\
\hline & & tension hold & & -1 & $5 \times 10^{-4}$ & $10 \mathrm{~min}$ & tension hold LCF \\
\hline \multirow{2}{*}{ IN718 } & \multirow{2}{*}{$\mathrm{LCF}$} & tension hold & \multirow{2}{*}{650} & -1 & $5 \times 10^{-4}$ & $10 \mathrm{~min}$ & tension hold LCF \\
\hline & & slow-slow & & -1 & $2 \times 10^{-4}$ & 0 & s-s LCF \\
\hline
\end{tabular}

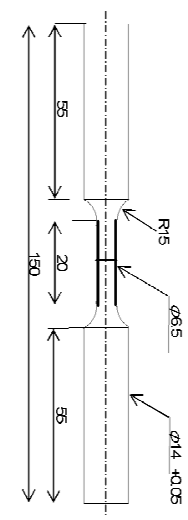

Fig.1 Geometry of fatigue specimen used.

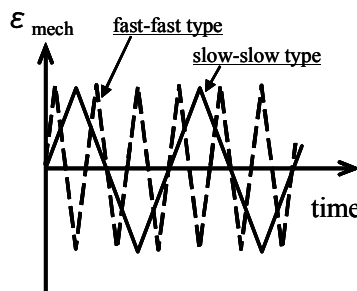

(a) Low cycle fatigue

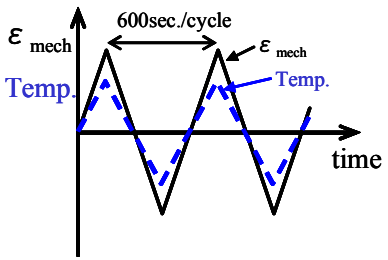

(c) IP TMF

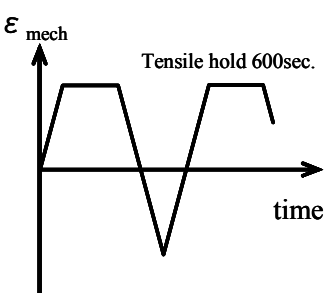

(b) Tension hold LCF

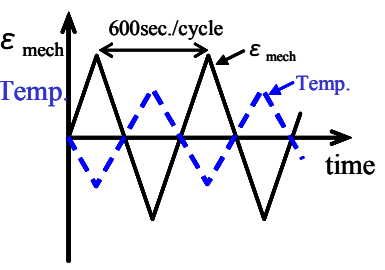

(d) OP TMF

Fig.2 Schematic illustration of the strain wave form in the LCF and TMF tests employed. 


\subsection{Low cycle fatigue (LCF) and thermo-mechanical fatigue (TMF) tests}

From the DFW joint, the solid cylindrical welded specimens were extracted as illustrated in Fig.1, where the welded interface was locating at the specimen center. For the comparison, cylindrical specimens of the base metals, IN718 and M247, were also prepared.

The LCF and TMF tests were carried out for both the welded joints and their base metals, according to the test program in Table 2. Here, the TMF tests were carried out such that the mechanical strain cycling is superimposed in a synchronized manner on the thermal free expansion strain cycling (see a schematic illustration in Fig.2). All tests were performed under strain-controlled condition in air, utilizing a servo-electro hydraulic test system. The tests for the DFW specimen were carried out such that the both IN718 and M247 shared a fifty-fifty length in the extensometer. The specimens were heated by a high frequency induction heating system. It has been confirmed by a preliminary test that the temperature difference between the shoulder part and the middle point of the specimen gauge section, were within $5^{\circ} \mathrm{C}$, when the specimens were heated at $650^{\circ} \mathrm{C}$. These temperature distributions were compensated during the natural cooling process in the TMF test. The test temperatures were represented and controlled through the $\mathrm{R}$ type thermocouples welded near the specimen shoulder part.

The isothermal LCF tests were conducted at $650^{\circ} \mathrm{C}$, where the experimental variables were strain range, strain rate and hold time introduced at the maximum tensile strain. The TMF tests were carried out under thermal cycling condition between $300-650^{\circ} \mathrm{C}$. Special attention was paid to the effect of temperature/strain phase angle, which was varied by two conditions: in-phase (IP; phase angle is $0^{\circ}$ ) and out-of-phase (OP; phase angle is $180^{\circ}$ ). The number of cycles to failure of the TMF and LCF lives was defined by the number of strain cycles at which the tensile stress was reduced by $25 \%$ from stationary value.

\section{Results}

The LCF and TMF lives of the DFW joint are summarized and compared with those of the base metals in Figs.3, 4 and 5. Note that the vertical axis of these figures denotes the mechanical strain range, $\Delta \varepsilon_{\text {mech }}$, in which thermal expansion strain is subtracted from the total strain in order to directly compare the TMF lives with the LCF ones. It is found from Figs.3, 4 and 5 that the lives of the dissimilar joints are significantly influenced by the test conditions and the loading modes. It is worth noting that the tension hold LCF lives of the DFW joint are significantly shorter than those under the other test conditions, and than those of the both base metals. Figure 6 schematically shows where and how the failures were taken place in the DFW joints under the respective test conditions and loading modes: failures under the f-f LCF and the TMF were occurred in the M247 side, while those under the s-s LCF and the tension hold LCF were taken place in the IN718 side near the interface. The details of the fatigue lives and the fracture behaviors will be shown hereinafter.

\subsection{Fatigue lives}

Now focus on the f-f LCF lives of the DFW joint and the base metals (Fig.3). The LCF lives of the DFW joint under f-f cycling are longer than those under s-s cycling; a usual trend in metallic materials. It is also found in Fig. 3 that the f-f LCF lives of the DFW joint are shorter than those of the two base metals $\left(\Delta \varepsilon_{\text {mech }}=1.0 \%\right)$, while the difference is not significant under lower strain ranges.

Figure 4 shows the tension hold LCF lives of the DFW joint and the base metals. To be compared, the f-f and s-s LCF lives of DFW joint were also plotted in Fig.4. As mentioned above, of particular importance is that the tension hold significantly reduces the fatigue lives compared with the f-f LCF and the s-s LCF. Furthermore, the lives of the joint are significantly shorter than those of both base metals. 


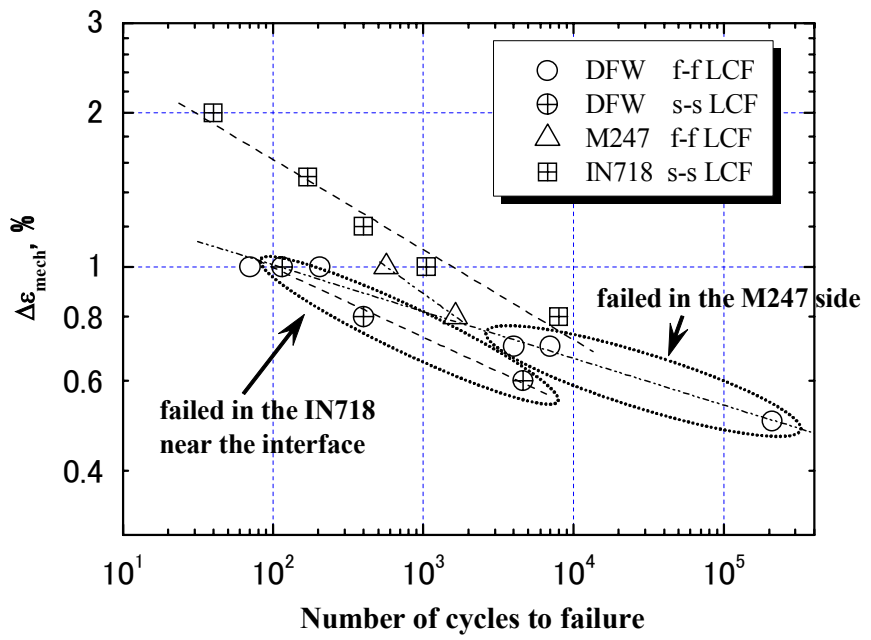

Fig.3 Strain-life relationship in the LCF tests for the DFW joint, the IN718 and the M247 base metals.

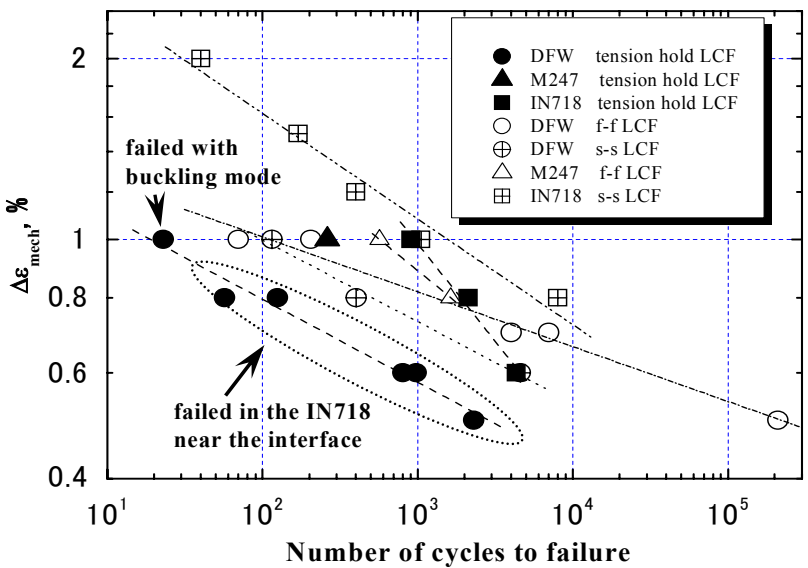

Fig.4 Strain-life relationship in the tension hold LCF tests. (DFW joint, the IN718 and the M247 base metals.)

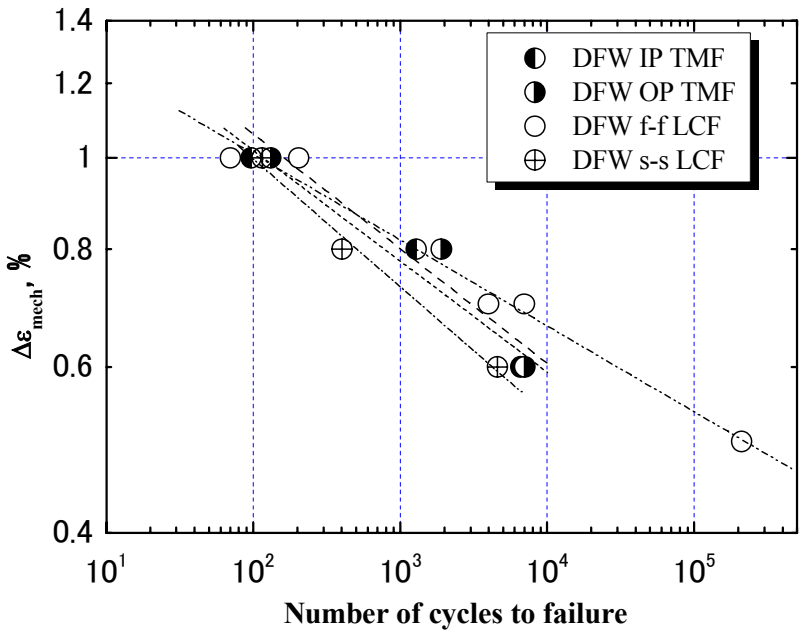

Fig.5 Strain-life relationship in the TMF tests for the DFW joint. 


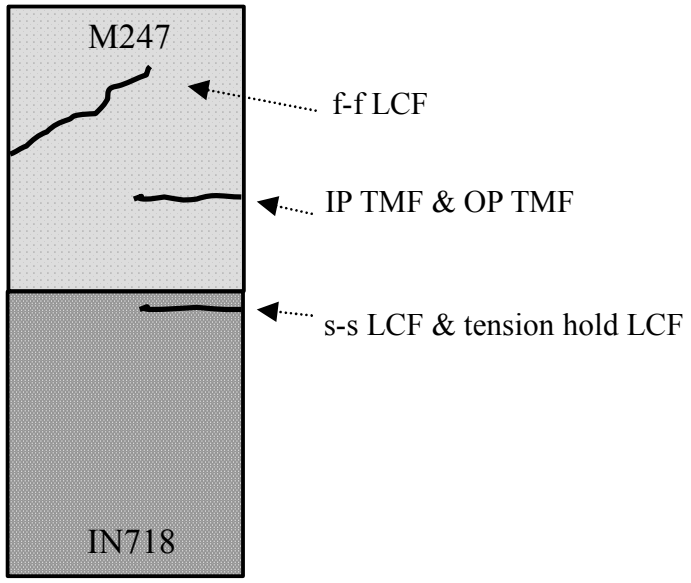

Fig.6 Schematic illustration showing the failure sites depending on loading modes.

Figure 5 shows the IP and OP TMF lives of the DFW joints comparing with the LCF lives of them. It is found from Fig.5 that the temperature/strain phase angles do not so significantly affect the TMF lives. It is also found that the TMF lives of the joints are almost comparable to the s-s type LCF lives. Thus, the following usual empirical law can be applied to the DFW specimen: the TMF lives under IP and OP conditions can be approximated by the LCF lives which are measured at the maximum and at the intermediate temperatures of the TMF tests, respectively ${ }^{(12)}$.

\subsection{Fracture behavior and fracture position in the DFW joint}

As shown in Fig.6, under the f-f LCF and the TMF conditions, all the fractures of the joints occurred on the M247 side apart from the weld interface. This result is not surprising, since the fatigue life of the M247 is shorter than that of the IN718 at the same strain range (see Fig.3). The fracture surfaces of the IP TMF condition are shown in Fig.7. It is found from Fig.7(b) that the fractures were occurred associated with the crack propagation along the dendritic boundaries in the M247. The fracture surfaces under the f-f LCF and the OP TMF revealed the almost similar characteristics in Fig.7.

On the contrary, under the tension hold LCF and the s-s LCF conditions, fractures were taken place in the IN718 side near the interface. Figure 8 shows the longitudinal cross section of the DFW joint failed under the tension hold LCF $\left(\Delta \varepsilon_{\text {mech }}=0.8 \%\right)$. It is found from

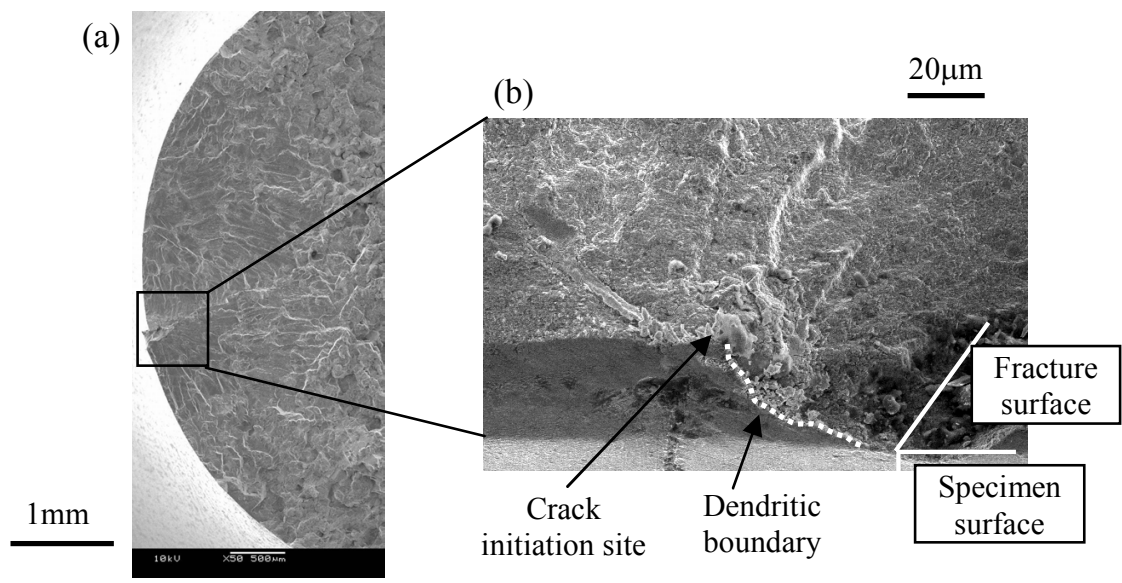

Fig. 7 Fracture surfaces of the DFW joints under the IP TMF loading. $\left(\Delta \varepsilon_{\text {mech }}=0.8 \%\right)$ 


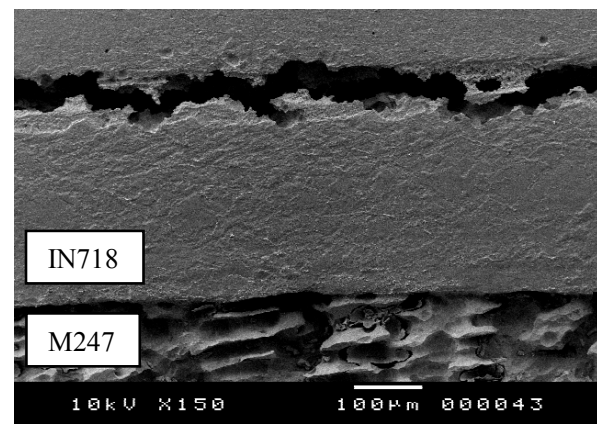

Fig. 8 Longitudinal cross section of the DFW specimen.(tension hold, $\Delta \varepsilon=0.8 \%$.)
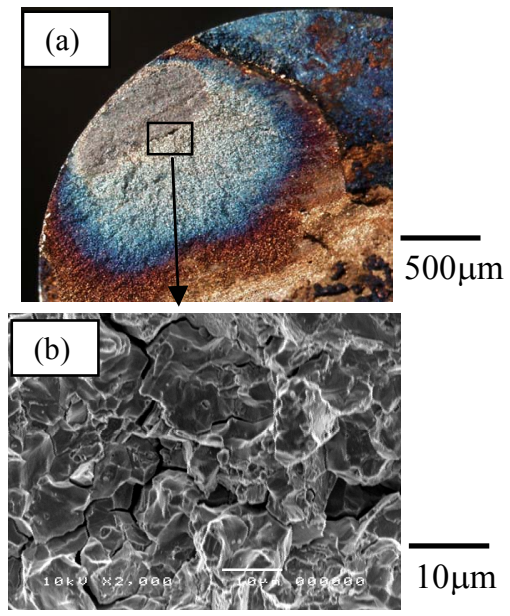

Fig.9 Fracture surface of DFW specimen. (tension hold LCF, $\Delta \varepsilon=0.5 \%$ )

Fig. 8 that the failures were taken place near the welded interface on IN718 side (about $300 \mu \mathrm{m}$ apart from the interface). Of particular importance is that the failure of the DFW joint was originated from the IN718 side and not from the M247, although the life of former base material was longer than that of the latter. This fracture pattern was seen in common independent on strain ranges. An exception was found under $\Delta \varepsilon_{\text {mech }}=1.0 \%$ where the failure occurred with specimen buckling on the M247 side.

A typical fracture surface under the tension hold LCF is given in Fig.9. The ellipsoidal mark seen in Fig.9(a) suggests that the crack initiated from inside of the specimen. Since the wedge-type intergranular fracture pattern is formed near the crack initiated position (see Fig.9(b)), the failure under this condition was dominated by creep damage.

\section{Discussion}

From the experimental results in Sec.3, it was found that the fatigue lives under the tension hold LCF were significantly shorter than those under the other test conditions, and than those of both base metals (see Fig.4). It was also found that the fracture behaviors under the s-s LCF and the tension hold LCF conditions were very unique: the failures occurred in the IN718 near the weld interface, although the IN718 has essentially longer life than the M247 base metal (see Fig.3; s-s lives of the IN718 are longer than the f-f LCF lives of M247). In this section discussion will be made on the effects of non-uniformity in mechanical properties of the two base metals and the inhomogeneity in the DFW joint on the failure mode and the fatigue life.

\subsection{Elastic follow-up in the DFW joint}

Figure 10 shows the change of stress range (Fig.10(a)) and that of stress ratio (Fig.10(b)) with the number of cycles during the tension hold LCF test. For an easier comparing, the stress ranges are normalized by those at the first strain cycle $(\mathrm{N}=1)$. It is found from Fig.10(a) that the stress range of the DFW joint is almost constant through the strain cycling, while the IN718 and the M247 monolithic specimen show the cyclic softening and hardening behavior, respectively. On the other hand, the stress ratio of the DFW joint continued to decrease with the cycles monotonically. It is worth noting that the stress ratio in the DFW joint is lower (larger in negative direction) than that of the IN718 base metal. These experiments strongly suggest that the progressive deformation may occur in the DFW joint.

Meanwhile the creep deformation resistance of IN718 is much lower than that of M247 at the present temperature regime; $650^{\circ} \mathrm{C}^{(13),(14)}$. This type of difference must be related to 
(a) Stress range

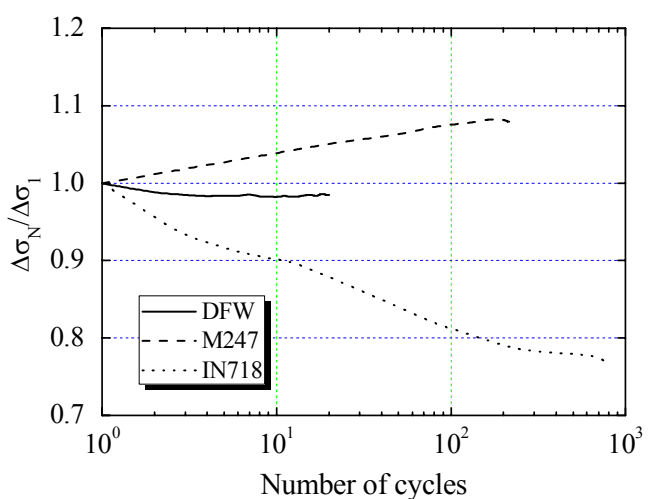

(b) Stress ratio

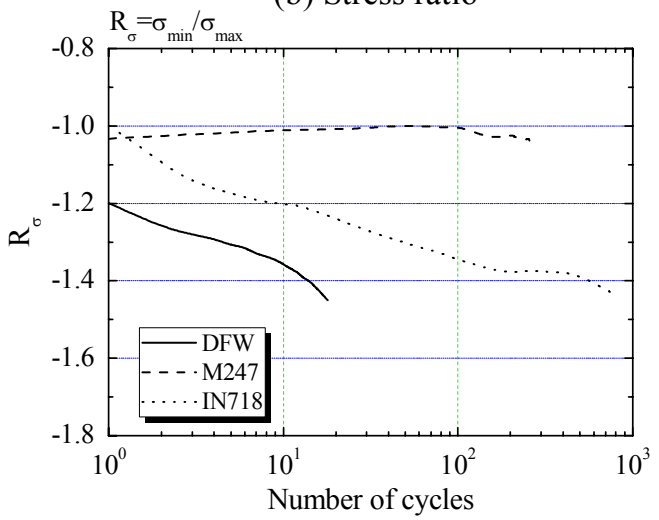

Fig.10 Variation of (a) the stress range and (b) the stress ratio with number of cycle. (DFW joint after LCF loading with $10 \mathrm{~min}$ tensile hold, $\Delta \varepsilon_{\text {mech }}=1.0 \%$ )

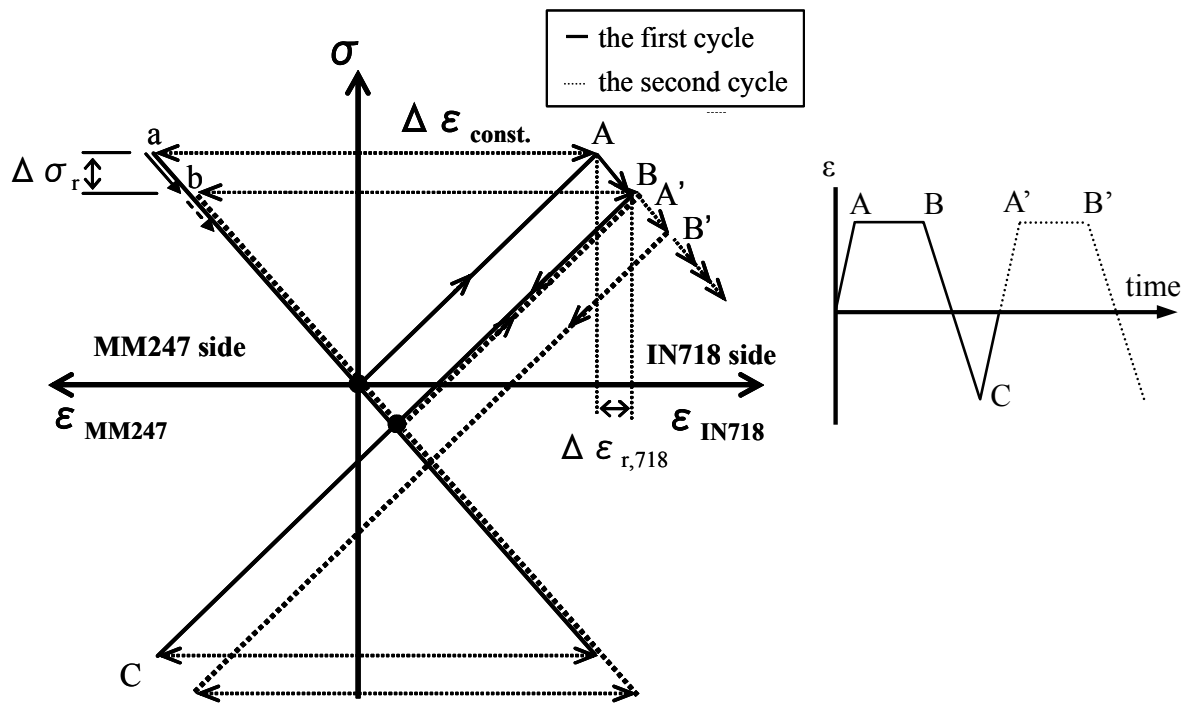

Fig.11 A schematic illustration of elastic follow-up mechanism in DFW joint under tensile hold LCF loading.

the specific characteristics in deformation and failure of the present DFW joint. Now consider a case in which the DFW joint is subjected to the strain-controlled LCF loading with tension hold time. One of the most possible inelastic deformation mechanisms is illustrated in Fig.11, where the right-half and the left-half express the strain level in the IN718 and the M247, respectively. Here, the IN718 and the M247 are assumed to be visco-elastic and elastic materials, respectively. During the tension hold period (A to B; see Fig.11), a load in the joint is relaxed by $\Delta \sigma_{r}$. During this hold time, the stress in the IN718 can be relaxed by creep deformation, while that in the M247 can be done only by elastic deformation. Through this mechanism a total strain of the DFW joint is kept constant on an appearance. However, this type of deformation leads to such an eccentric creep deformation (by an amount of $\Delta \varepsilon_{r, 718}$ in Fig.11) on IN718 side. During the reversed loading process period (B to $\mathrm{C}$ in Fig.11), the stress is unloaded and relaxed elastically in the both materials. From the next strain cycle period ( $\mathrm{A}^{\prime}$ to $\mathrm{B}^{\prime}$ in Fig.11), however, a tensile creep strain is again accumulated on the IN718 side. Thus the creep damage can be evolved only in the IN718, which will be greater than the damage in the monolithic IN718 specimen.

Comparing Fig. 11 with the experimental results, some reasonable agreements are seen. First of all, the elastic follow-up mechanism can estimate the failure site on the In718. Additionally, this mechanism provides such an estimation that the stress ratio may change 
in a monotonic manner during the tension hold LCF test cycling. This estimation is reasonably realized in Fig.10(b). An experimental result that the DFW failed accompanying with buckling mode under $\Delta \varepsilon_{\text {mech }}=1.0 \%$ (see Fig.4), can be also understood naturally, by the mechanism.

\subsection{Effect of inhomogeneous microstructure}

Figure 12 expresses the microstructures near the weld interface after the tension hold LCF test. Note that the failure was near but not just at the prior interface. In order to explore a microstructural aspect on the failure position, an attention was paid to the grain size distribution: see some marks circled by bright lines in Fig.12. It is found that the grain size in IN718 changes depending on the distance from the prior interface: i.e.; the grain size near the interface is relatively large (about $50-10 \mu \mathrm{m}$ ), while that near the crack propagation region is small about $5-10 \mu \mathrm{m}$.

Figure 13 shows the hardness distribution along the corresponding heat affected zone (HAZ). The hardness of the HAZ seems significantly lower than that of the base metal. In general weldments, it has been well recognized that a degree of stress triaxiality is promoted in soft zone (; lower hardness) and that creep cavitation can be easily originated there ${ }^{[15]}$. Note that the hardness near the interface is lower than those of the base metals. Thus, the mechanical factor or a promotion of stress triaxiality must significantly reduce the DFW life

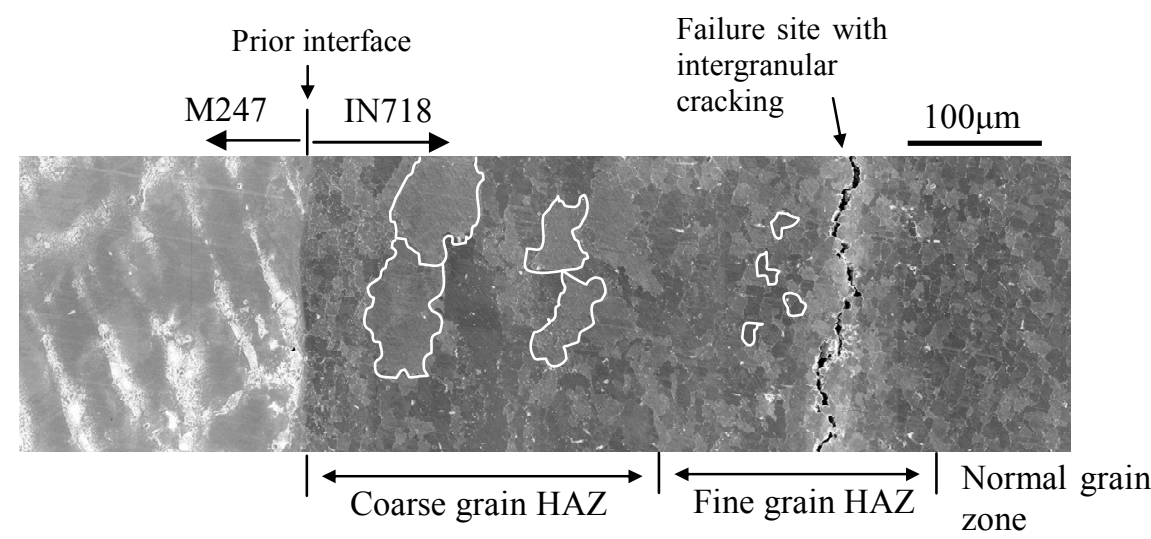

Fig.12 Microstructure of IN718 near the interface after the tension hold LCF test. Grain size near the interface is about $50-100 \mu \mathrm{m}$, while that apart from the interface is about $10-20 \mu \mathrm{m}$.

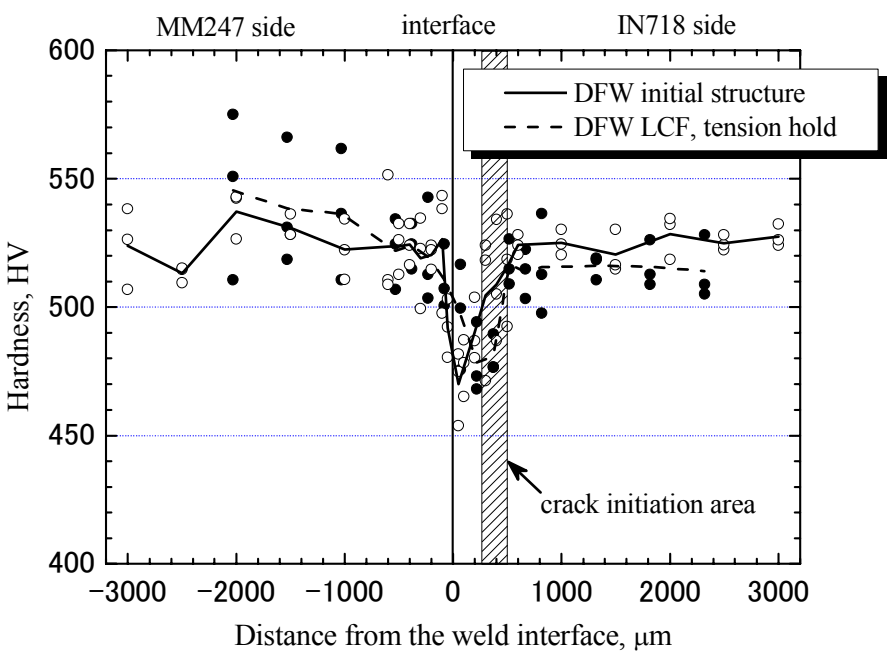

Fig.13 Hardness test result of DFW after the tension hold LCF tests. 
with tension hold time, in a synchronized manner with microstructural inhomogeneity as shown in Fig. 12

\section{Summary}

In this work, dissimilar superalloys welded joint between the cast polycrystalline Mar -M247 and the forged Inconel 718 alloys (DFW joint) has been fabricated by friction welding. The high temperature fatigue strength, low cycle fatigue and thermo-mechanical fatigue of the joint, were quantified, compared with those of the base metals. It was shown the lives and failure positions of the dissimilar joints were significantly influenced by the test conditions and loading modes, which might be depending on both the macroscopic difference of deformation behavior between two base metals and the promotion of stress triaxiality induced by the inhomogeneous microstructure in the DFW joint. Especially, an elastic follow-up was shown to be an important mechanism to be concerned for considering the creep/fatigue failure.

\section{Acknowledgment}

Financial support by Grant-in-aid from the Ministry of Education, Japan (No. 19860036) is greatly acknowledged.

\section{References}

(1) M. Preuss, J.W.L Pang, P.J. Withers, G.J. Baxter, Metall. Mater. Trans., Vol.33A, (2002), pp.3215-3126.

(2) M. Preuss, J. Quinta da Fonseca, I. Kyriakoglou, P.J. Withers, G.J. Baxter, Superalloy 2004, TMS, $10^{\text {th }}$ Int. Symposium, Seven Springs, Champion, PA, (2004), pp.477-484.

(3) P. Adam, Welding of high strength gas turbine alloys, Applied Science Publisher Ltd, (1978), pp.737-768.

(4) C.T. Sims, W.C. Hagel, The Superalloys, John Wiley, New York, (1972), pp.509-532.

(5) M. Preuss, J.W.L Pang, P.J.Withers, G.J. Baxter, Metall. Mater. Trans., Vol.33A, (2002), pp.3127-3134.

(6) M. Preuss, P.J. Withers, G.J. Baxter, Mater. Sci. Eng., Vol. A437, (2006), pp.38-45.

(7) M. Okazaki, Y. Mutoh, T. Yada, M. Yamaguchi, J. Soc. Mater. Sci. Vol.36, No.413, (1987), pp.1239-1245.

(8) M. Sakaguchi, M. Okazaki, JSME international, Vol.49A, No,3, (2006), pp.345-354.

(9) M. Sakaguchi, M. Okazaki, Int. J. Fatigue, Vol.29, No.9, (2007), pp.1959-1965.

(10) M. Okazaki, M. Sakaguchi, Int. J. fatigue, Vol.30, No.2, (2008), pp.318-322.

(11) K. Kuwabara, A. Nitta, T. Kitamura, J. Soc. Mater. Sci., Vol.32, No.357, (1983), pp.657-661. (in Japanese)

(12) S. Taira, M. Fujino, R. Ohtani, Fatigue Eng. Mater. Struc., Vol.1, (1979), pp.495-500.

(13) C.R. Brinkman, M.K. Booker, J.L. Ding, Superalloys 718, 625 and Various Derivatives, TMS, (1991), pp.519-528.

(14) M.V. Nathal, R.D. Maier. L.J. Ebert, Metal. Trans. A, Vol.13A, (1982), pp.1767-1774.

(15) T.H. Hyde, A.A. Becker, Int. J. Press. Vess. \& Piping, Vol.78, (2001), pp.765-773. 